\title{
Evaluation of 52 patients with Guillain Barré syndrome between 2015 and 2019 in Kocaeli, Turkey - An epidemiological study
}

\author{
Buket Özkara, Yiğit Can Güldiken, Faik Budak, Özge Demirkol, Sedat Gül \\ Department of Neurology, Faculty of Medicine, Kocaeli University, Turkey
}

\begin{abstract}
Purpose. The primary purpose of this study is to evaluate the demographic, clinical and laboratory findings of patients with Guillain Barré syndrome (GBS), assess the clinical course, treatment outcomes and introduce GBS subgroups in Kocaeli, Turkey.

Methods. Patients admitted to Kocaeli University Neurology Clinic between 2015 and 2019 with a preliminary diagnosis of autoimmune inflammatory acute polyneuropathy were included. Demographic, clinical, laboratory data have been collected and analyzed prospectively. Prognosis was evaluated according to the sum of total scores from GBS disability scoring and Medical Research Council (MRC) scoring guidelines and the need for mechanical ventilation. For statistical analysis, IBM SPSS 20.0 (SPSS Inc., Chicago, IL, USA) was used.

Results. 52 GBS patients were evaluated. Mean age at initial presentation was $50.33 \pm 17.2 .57 .7 \%$ of the patients reported a recent disease, most common one being upper respiratory tract infection (URTI). Progression of the disease was reported as ascending in 39 patients $(75 \%)$ and descending in $13(25 \%)$. Muscle weakness was symmetrical in 46 patients $(88.4 \%)$ and asymmetrical in $6(11.6 \%)$. 30 out of 52 patients $(57.6 \%)$ had an elevated erythrocyte sedimentation rate (ESR), $40(76.9 \%)$ had leukocytosis, $11(21.1 \%)$ had hyponatremia. Cerebrospinal fluid analysis of 39 patients revealed elevated protein levels in 32 of them $(61.5 \%) .41$ patients $(78.8 \%)$ were treated with IVIG, $8(15.4 \%)$ with plasmapheresis and IVIG, 2 patients $(3.8 \%)$ were treated with plasmapheresis only. Conclusion. Our study revealed both consistent and contradicting findings to the literature that may lead further debate/research on the epidemiology of GBS.
\end{abstract}

Keywords: Guillain Barré syndrome, acute inflammatory demyelinating polyneuropathy (AIDP), acute motor axonal neuropathy (AMAN), acute motor-sensory axonal neuropathy (AMSAN), Miller-Fisher syndrome (MFS)

\section{INTRODUCTION}

Guillain Barré syndrome (GBS) is an acute acquired polyradiculoneuropathy that presents with progressive flask paralysis, cranial nerve manifestations and sensory, motor and autonomic deficits $(1,3,4)$. The diagnosis is usually based on clinical criteria, however, nerve conduction alterations on electromyography (EMG) and albuminocytologic dissociation on cerebrospinal fluid (CSF) analysis also supports diagnosis of GBS $(1,5)$. Rapidly progressive, ascending, generalized weakness and areflexia are the main signs and symptoms seen in most cases $(1,7)$. Sensory deficits are usually less significant. The severity of symptoms and dissem- ination of neuropathy can vary from mild patients who can be easily mobilized without any additional support to severe tetraplegia $(9,10)$. Regarding the electrophysiological alterations and immunological mechanisms, GBS has been divided into different clinical subtypes such as: acute inflammatory demyelinating polyneuropathy (AIDP), acute motor axonal neuropathy (AMAN), acute motor-sensory axonal neuropathy (AMSAN) and Miller-Fisher syndrome (MFS) $(3,10)$.

\section{AIM}

The purpose of this study is to evaluate the demographic, clinical and laboratory findings of the 
patients who had been hospitalized and followed up with a preliminary diagnosis of autoimmune inflammatory acute polyneuropathy, follow-up their clinical course, treatment and prognosis as well as the determination of common GBS subgroups.

\section{MATERIALS AND METHODS}

52 patients who were admitted to Kocaeli University Neurology Clinic between 2015 and 2019 were included. Their demographic, clinical, laboratory data have been collected and analyzed prospectively.

Inclusion criteria:

1. Acute presentation (up to 4 weeks), progressive, bilateral muscle weakness

2. Accompanying sensory findings

3. Significant decrease or loss in deep tendon reflexes

4. Typical electrophysiological findings

5. Laboratory confirmation

6. Exclusion of other neurological diseases which have been included in the differential diagnosis

7. No significant level of sensory deficiency

8. Patients who are older than 18 years of age

Patients were classified as AIDP, AMAN, AMSAN and MFS according to their clinical presentation and electrophysiological findings.

Prognosis was evaluated according to the sum of total scores from GBS disability scoring and Medical Research Council (MRC) scoring guidelines and the need for mechanical ventilation.

For statistical analysis, IBM SPSS 20.0 (SPSS Inc., Chicago, IL, USA) was used. KolmogorovSmirnov test was used to assess normal distribution. Numerical variables were assigned as mean \pm standard error and median $\left(25^{\text {th }}\right.$ percentile $-75^{\text {th }}$ percentile) and frequency (percentiles). Differences between the groups were determined by Student $\mathrm{T}$ Test in numeric variables with normal distribution; by Mann Whitney U Test in numeric variables that don't follow normal distribution and by Yates Chi Square analyses in categorical variables. $\mathrm{P}$ value was $<0.05$ and results were accepted as statistically significant.

All of the patients who were participated in this research have given informed consent. The patients were informed about the usage of the data and possible implications. Consents were obtained after the treatment and follow up period was completed.

\section{RESULTS}

52 GBS patients were evaluated. Mean age at the initial presentation was $50.33 \pm 17.2$. Bimodal distribution pattern with a large peak at $3^{\text {rd }}$ decade and a smaller peak in $5^{\text {th }}$ decade was detected. Male to female ratio was 1.16/1. Seasonal distribution showed that $42.3 \%$ of our patients presented in winter. $57.7 \%$ of our patients reported a recent disease, most common one being upper respiratory tract infection (URTI) in $28.8 \%$ of the patients. Diarrhea in 11 patients $(21.2 \%)$, cancer in 2 patients $(3.8 \%)$ and urinary tract infection (UTI) in 2 patients $(3.8 \%)$ were also detected. In 29 patients out of 52, prodromal findings were evident before initial motor symptoms. 16 patients $(30.8 \%)$ had paresthesia in hands and feet, 8 patients $(15.3 \%)$ had aching of the legs and 5 patients $(9.6 \%)$ had back and low back pain as prodromal symptoms. Progression of the disease was ascending type in 39 patients $(75 \%)$ and descending type in 13 patients $(25 \%)$. Muscle weakness was symmetrical in 46 patients $(88.4 \%)$ and asymmetrical in 6 patients (11.6\%). 21 out of 52 patients had cranial nerve involvement. 5 of these patients $(9.6 \%)$ had facial nerve involvement, 6 of them $(11.5 \%)$ had bulbar and 6 of them (11.5\%) had ocular involvement. 4 of them (7.6\%) had both fascial and bulbar involvement. 20 patients $(38.4 \%)$ had autonomic involvement, 5 of them had hypotension, 4 of them had urinary retention and 11 of them had tachycardia. Additionally, respiratory muscles were affected in 11 patients $(21.1 \%), 7$ patients $(13.5 \%)$ needed intensive care unit admission and 2 patients died.

Laboratory findings revealed that 30 out of 52 patients $(57.6 \%)$ had an elevated erythrocyte sedimentation rate (ESR), 40 patients $(76.9 \%)$ had leukocytosis, 11 patients $(21.1 \%)$ had hyponatremia. Cerebrospinal fluid samples which have been obtained from 39 patients revealed that protein levels were elevated in 32 of them $(61.5 \%)$ and measured within the normal range for 7 of our patients $(13.5 \%)$.

During hospitalization, 41 patients $(78.8 \%)$ were treated with IVIG, 8 patients $(15.4 \%)$ were 
treated with plasmapheresis and IVIG, and 2 patients $(3.8 \%)$ were treated with plasmapheresis only. 1 patient was managed with careful observation due to mild symptoms.

To further analyze the results, our patient group was divided regarding the age of 50 years. Hughes scores were significantly lower in patients younger than $50(\mathrm{p}=0.001)$. Among 25 patients who were younger than 50, 2 patients recovered completely without any sequel, 11 patients recovered with mild sequels (MRC score: 50-60) and 11 patients recovered with moderate sequels (MRC score: 30-40) and 1 patient recovered with severe sequels. Among 27 patients who were older than 50 years of age, 2 of them recovered completely without any sequel, 2 patients recovered with mild sequels (MRC score: 50-60), 18 patients recovered with moderate sequels (MRC score: $30-40$ ), 3 patients recovered with severe sequels and 2 patients died. There were no significant difference between these two groups regarding the need for a mechanical ventilation $(p=0.101)$. When comparing the male and female patients, there were no significant difference in MRC scores, Hughes scores and the need for mechanical ventilation $(p=0.6)$. Comparing the patients with predisposing diseases and patients without predisposing disease, there were no significant difference in MRC scores, Hughes scores and the need for mechanical ventilation $(p=0.9)$. There was no significant difference for prognosis between the patient groups with and without prodrome findings.

There were no significant statistical difference between the ascending and descending types of the disease and the symmetrical and asymmetrical involvement when comparing by their MRC, Hughes scores $(p=0.6)$ and the need for mechanical ventilation $(p=0.5)$. Patients with bulbar involvement had significantly lower MRC scores and higher Hughes scores (mean: 4.0). 19 patients who had autonomic involvement also had significantly higher Hughes scores. Patients with respiratory muscle involvement had statistically significant lower MRC and higher Hughes scores. Comparing the 41 patient who only received IVIG therapy to the 8 patients who received both IVIG and plasmapheresis therapy regarding their need for mechanical ventilation, no significant difference was noted.

52 patients with GBS were divided into subgroups regarding their electrophysiological find- ings. 25 patients (48.1\%) were classified as AIDP, $8(15.4 \%)$ were classified as AMAN, $13(25.0 \%)$ were classified as AMSAN and $6(11.5 \%)$ were classified as MFS. These subgroups were evaluated for predominant predisposing diseases. For 25 AIDP patients, 7 had upper respiratory tract infection, 4 had diarrhea and 1 had urinary tract infection. Among 8 patients in AMAN subgroup, 2 had upper respiratory tract infection and 2 had diarrhea. Among 13 patients in AMSAN subgroup, 3 patients had upper respiratory tract infection, 3 had diarrhea, 2 had cancer and 1 had urinary tract infection. Lastly, among 6 patients in MF subgroup, 3 had upper respiratory tract infection and 2 had diarrhea. The 2 patients whose stool cultures were positive for $C$. jejuni were in AMSAN subgroup. AMSAN and AIDP subgroups have a higher rate of prodrome symptoms. Symmetric involvement was more common in all GBS subgroups. Descending progress was more common in AMAN and AMSAN groups compared to others. There was no difference between the groups in terms of other examination findings (symmetric asymmetric progression, motor and sensory findings). No significant difference was seen between AIDP and AMSAN groups in terms of cranial nerve involvement. No cranial nerve involvement was seen in AMAN group. All patients with MFS had ocular cranial nerve involvement. Autonomic involvement was more common in patients with axonal involvement, especially in the AMSAN subgroup. 2 of the patients in AIDP subgroup and 3 of the patients in AMSAN subgroup needed mechanical ventilation due to respiratory muscle involvement. Findings of autonomic involvement such as hypotension, tachycardia, arrhythmia and urinary retention were seen in 8 out of 25 patients in AIDP subgroup, 2 out of 6 patients in AMAN subgroup and 10 out of 13 patients in AMSAN group. None of the patients with MFS had autonomic involvement. 4 patients out of 25 in AIDP subgroup and 6 patients out of 13 in AMSAN group had respiratory muscle involvement. Respiratory muscle involvement was more common in AMSAN subgroup.

There were no statistically significant difference between subgroups in terms of the laboratory findings such as serum sodium level, serum leukocyte count, erythrocyte sedimentation rate and the presence of albuminocytologic dissociation in 
cerebrospinal fluid. Pre-treatment mean MRC score was 45.5 and mean Hughes score was 2.8 in AIDP subgroup while post-treatment mean MRC score was 54.4 and mean Hughes score was 1.8 . Pre-treatment mean MRC score was 42.7 and mean Hughes score was 3 in AMAN subgroup while post-treatment mean MRC score was 54.4 and mean Hughes score was 1.5. Pre-treatment mean MRC score was 34.9 and mean Hughes score was 4.08 in AMSAN subgroup while post-treatment mean MRC score was 50,6 and mean Hughes score was 2.7. Pre-treatment mean MRC score was 57 and mean Hughes score was 1.17 in MFS subgroup while post-treatment mean MRC score was 58.6 and mean Hughes score was 0.33. GBS disability score (Hughes) was detected to be higher and pre-treatment MRC score was detected to be lower in axonal variants.

\section{DISCUSSION}

Guillain-Barré syndrome (GBS) is an autoimmune, often severe and fulminant polyradiculoneuropathy with acute onset. Approximately 100,000 people worldwide have been affected each year. GBS appears as the most common cause of acute and subacute flaccid paralysis since poliomyelitis is eradicated from a large part of the world $(1,6)$. Its incidence in a year is $0.4-2.6$ per 100,000 $(6,7)$. GBS is considered among the most common causes of neurological diseases requiring referral to intensive care units (ICU) $(2,12)$. Although it is not frequently seen in the community, early diagnosis and treatment is crucial in terms of the prognosis of the disease.

In previous studies, there are reports indicating gender and age difference in GBS cases $(9,16)$. With advancing age, there is an increase in the incidence of the disease which may be also classified as directly proportional. This increase is consistent with decreased immune-suppressing mechanisms and consequently increased susceptibility to autoimmune diseases in the elderly. Age distribution of the patients is between 8 months and 81 years old in the literature, with the ages between 50 and 74 years experiencing the maximum number of attacks. In our study, the peak incidence was seen in the 3rd decade, with a similar peak in the 5 th decade as well. Men are more often affected by the disease than women, and in the literature the rate is $1.25 / 1$. In our study, the male / female ratio was found to be $1.16 / 1$. It was also seen more frequently in men than seen in women, but the difference between genders has been found out to be lower. GBS is considered as a sporadic disease that does not show seasonal clustering. Seasonal fluctuations are likely to be associated with changes in infectious diseases, but these observations are rarely statistically significant $(12,13)$. Unlike the literature, our study showed a tendency to accumulate in winter months. Preceding infection rate in GBS has been reported between 46 and 70\% $(8,15)$. In our study, consistent with the former studies, $57.7 \%$ of patients had a history of preceding infection within the previous month. $C$. jejuni is the dominant infection found in $25-50 \%$ of adult GBS patients and more common in Asian countries (14). In 5 of our patients, Campylobacter jejuni culture was positive in stool (9.6\%). Tingling and dysesthesia in extremities are common prodromal signs in GBS cases and the lower extremities are affected more in $50 \%$ of individuals $(14,16)$. In our study, lower extremity involvement was higher as it was stated in the corresponding literature. In typical Guillain-Barré syndrome, rapidly progressing symmetrical bilateral loss of strength is the key symptom in most patients. The loss of muscle strength typically starts from the distal parts of lower extremities and ascends as the disease progresses. However, an onset originating from the upper limbs, continuing with a descend as the disease progresses and an asymmetrical involvement may also be seen $(2,10)$. In literature, these findings have not been directly linked to prognosis (11-13). In our study, there were no significant differences between disease output when considering the onset of the disease as ascending-descending, symmetrical-asymmetrical and evaluating with the prognostic factors such as the MRC, Hughes scores and mechanical ventilation need. In previous studies, autonomic nervous system involvement has been reported between $10-45 \%(1,4)$. In our study, there were signs of autonomic nervous system involvement (hypertension, tachycardia, cardiac arrhythmia, urinary retention) in $38.4 \%$ of patients, consistent with the previous studies. Autonomic involvement has been associated with poor prognosis in previous studies $(2,16)$. In our study, it was found that patients with autonomic involvement had a greater need for 
mechanical ventilation. In the literature, it has been stated that respiratory muscle involvement is present in $20-30 \%$ of patients $(1,15)$. In our study, respiratory muscle involvement was observed in $21.2 \%$ of the patients, in accordance with the literature. In the literature, it has been reported that $30-35 \%$ of GBS patients have hyponatremia and these patients have a poor prognosis $(2,3,8)$. In our study, hyponatremia rate $(21 \%)$ was lower than the literature, however no significant relation to poor prognosis have been identified. In the literature, elevation in erythrocyte sedimentation rate (ESR) and serum leukocyte levels have been reported in $50 \%-60 \%$ of GBS patients $(8,9)$. In our study, patients with an increased ESR (57.6\%) was found to be compatible with the literature. Leukocytosis was detected in 6 of our patients (11.5\%). Leukocyte values of 40 patients were normal (76.9\%). Six of our patients had leukopenia $(11.5 \%)$. The rate of leukocytosis found in our study was lower than the literature $(4,13)$.

Some reports have suggested that increased ESR and leukocytosis are associated with poor prognosis $(3,10)$. The detection of increased ESR and elevated serum leukocyte levels in patients with poor prognosis was thought to be related to the severity of the prior infection and the degree of inflammation (11). However, in our study, no significant association was found between ESR, serum leukocyte levels and the prognosis. In $80 \%$ of GBS patients, albuminocytologic dissociation is observed in the CSF. In our study, from the total 39 patients' that CSF examination could have been performed; a high protein level in CSF was observed in 32 patients $(61.5 \%)$ and normal CSF protein levels in 7 of our patients (13.5\%). Many randomized controlled trials have been conducted to examine the advantages of immunotherapy in Guillain-Barré syndrome. Intravenous immunoglobulin (IVIG) and therapeutic plasma exchange (TPE) are proven to be effective $(3,13,14)$. In studies comparing IVIG and plasmapheresis treatments in GBS, no superiority was detected to each other $(3,8)$. The combination of IVIG after TPE was not found significantly effective when compared to TPE or IVIG alone. In our study, no significant difference was found when improvement in GBS scores of patients receiving IVIG only and patients receiving IVIG and TPE are compared.

The frequency of GBS subtypes varies in the world. The possible cause of this is thought to be the environmental factors, the agents causing the primary infection and the differences in immune response as well as the geographical variability (9). In Europe and North America, acute inflammatory demyelinating polyneuropathy (AIDP) accounts for more than $90 \%$ of GBS cases. The prevalence of acute motor axonal neuropathy (AMAN) subtype in Europe and North America was found to be 4\% $(9,19)$. However, in Asia, Central and South America, AIDP is seen on an average of $55 \%$, whereas axonal variants of GBS, for example, AMAN and acute motor sensory axonal neuropathy (AMSAN) are found to represent 30$47 \%$ of total cases. In Western countries, Miller-Fisher syndrome (MFS) accounts for $5-10 \%$ of all GBS cases, this rate increases in east Asia and reaches $25 \%$ in Japan $(9,17)$. In our study, we determined the prevalence of AIDP as $48.1 \%$, AMAN 15.4\%, AMSAN 25\%, and MFS 14.5\%. We found that the prevalence of AIDP in our study was lower when compared to Europe and similar to Asia, Central and South America. The prevalence of axonal forms (AMAN + AMSAN) in our study (29.4\%) was found higher than Europe, and lower than Asia, Central and South America. This situation can be explained by our country's geographic, socioeconomic and cultural location in a spectrum between the western and eastern societies. It was seen that the prevalence of MFS was higher than that of European countries. The data on seasonal distribution, prodromal symptoms, concomitant diseases, examination findings, autonomic involvement, and laboratory findings of GBS variants are insufficient in the literature. It is reported that $C$.jejuni enteritis is seen in $30-40 \%$ of axonal forms as the primary disease $(11,16)$. In our study, when the groups are compared according to the primary disease, it was observed that the rate of URTI (27.7\%) in the AIDP group was higher than the other groups.

There were no statistically significant difference in GBS subgroups in terms of laboratory findings (serum sodium, leukocyte levels and ESR) and the presence of albuminocytologic dissociation in the CSF. Campylobacter jejuni was detected positive in stool culture of two patients diagnosed with AMSAN.

In different clinical subtypes of GBS, different patterns can be observed as well as similar signs and symptoms. It has been reported that especially 
axonal forms show a faster and more severe course with more cranial nerve involvement and need for mechanical ventilation $(9,17)$. In our study, autonomic involvement and mechanical ventilator requirement was greater in axonal involvement (especially in the group diagnosed with AMSAN).

IVIG or IVIG + TPE treatments did not differ in prognosis for all GBS subgroups.

Although the AMAN form of GBS is traditionally associated with poor clinical prognosis, it has been demonstrated that AMAN patients can also recover rapidly $(9,10)$. In our study, similarly, axonal variants were found to have high GBS disability (Hughes) scores and low MRC scores on admission, but when examined for improvement in both GBS disability scores and MRC scores, it was found that axonal variants improved better than the demyelinating variant at the end of six months. Considering the expected delay in nerve regeneration following an axon damage, this rapid healing pattern seems surprising. This may be explained by early diagnosis of patients, prompt access to treatment options (IVIG, TPE), responding the need for mechanical ventilators and providing adequate supportive therapy. Although the mechanism is not clearly defined in axonal forms, it is thought that antiganglioside antibodies (anti GM1)

\section{REFERENCES}

1. Van Doorn PA, Ruts $L$, Jacobs BC. Clinical features, pathogenesis, and treatment of Guillain-Barré syndrome. Lancet Neurol. 2008; 7:939-950.

2. González-Suárez I, Sanz-Gallego, Rivera FJR, et al. Guillain-Barré Syndrome: Natural history and prognostic factors: a retrospective review of 106 cases. BMC Neurology. 2013;21:13-95.

3. Burns TM. Guillain-Barré syndrome. Semin Neurol. 2008 Apr;28(2):152-67.

4. Sejvar JJ, Baughman AL, Wise M, et al. Population incidence of Guillain Barré syndrome: a systematic review and meta-analysis. Neuroepidemiology. 2011;36:123e33.

5. Newswanger DL, Warren CW. Guillain Barré Syndrome. American Family Pysician. 2004;9(10).

6. Czaplinski A, Steck AJ. Immune mediated neuropathies. J Neurol. 2004;251:127-137.

7. Italian Guillain-Barré Study Group. The prognosis and main prognostic indicators of Guillain Barré syndrome. A multicentre prospective study of 297 patients. Brain. 1996;119:2053.

8. Clinical E, Neurology EP. Guillain-Barré syndrome variants in Emilia-Romagna, Italy, 1992-3: incidence, clinical features, and prognosis. Emilia-Romagna Study Group on Clinical and Epidemiological Problems in Neurology. J Neurol Neurosurg Psychiatry. 1998 Aug;65(2):218-24.

9. Akbayram S, Doğan M, Akgün C, Peker E, Sayın R, Aktar F, Bektaş MS, Caksen $\mathrm{H}$. Clinical features and prognosis with Guillain-Barré syndrome. Ann Indian Acad Neurol. 2011 Apr;14(2):98-102. and complement products cause temporary nerve conduction loss due to inactivation of voltage-gated sodium channels $(9,11,17)$. It has been reported that focal axonal membrane damage may occur as a result of this temporary inactivation (17). The severity of the local immune reaction may lead to reversible conduction failure or axonal degeneration. If the autoimmune attack is limited in a small area and can be treated early, nodal function may improve rapidly and axonal degeneration is not observed $(9,17)$. Therefore, early diagnosis and treatment of these patients is crucial.

\section{CONCLUSIONS}

With respect to the information stated above, it can be concluded that the patients with acute axonal damage, electrophysiological conduction block and those received prompt treatment can have a better prognosis.

As a result, we think that with a study that is including a greater number of patients, we would have more data on the subtypes of GBS, clinical severity, treatment responses and disease outcomes that are seen in our country. However, this study may contribute greatly to meta-analysis in the future.

10. Simovic $D$. Autoimmune neuropathies and treatment with intravenous immunoglobulins. Therapy. 2005;2(5):797-80.

11. Arami MA, Yazdchi M, Khandaghi R. Epidemiology and characteristics of Guillain-Barré syndrome in the northwest of Iran. Ann Saudi Med. 2006 Jan-Feb;26(1):22-7.

12. Ress JH, Thompson RD, Smetton NC, Hughes RAC. An epidemiological study of Guillain-Barré syndrome in southeast England. $J$ Neurol Neurosurg Psychiatry. 1998;64:74-77.

13. Cheng BC, Chang WN, Chang CS, et al. Guillain-Barré syndrome in southern Taiwan: clinical features, prognostic factors and therapeutic outcomes. Eur J Neurol. 2003;10:655-662.

14. Chio A, Cocito D, Leone M, Giordana MT, Mora G, Mutani R. Guillain Barré syndrome: a prospective, population-based incidence and outcome survey. Neurology. 2003;60:1146-1150.

15. Willison $\mathrm{HJ}$, Jacobs $B C$, van Doorn PA. Guillain-Barré syndrome. Lancet. 2016 Aug 13;388(10045):717-27.

16. Chowdhury D, Arora A. Axonal Guillain-Barré syndrome: a critical review. Acta Neurol Scand. 2001;103(5):267-277.

17. Sudulagunta SR, Sodalagunta MB, Sepehrar M, Khorram H, Bangalore Raja SK, Kothandapani S, Noroozpour Z, Aheta Sham M, Prasad N, Sunny SP, Mohammed MD, Gangadharappa R, Nidsale Sudarshan R. Guillain-Barré syndrome: clinical profile and management. Ger Med Sci. 2015 Sep 21;13. 